\title{
Porcine carcass phantom for ultrasound imaging and hands-on practice in needle insertion in the thoracic paravertebral space
}

\author{
Faraj W. Abdallah, MD • Graham Hocking, FANZCA • \\ Vincent W. Chan, MD
}

Received: 21 May 2011/ Accepted: 14 June 2011/Published online: 7 July 2011

(C) Canadian Anesthesiologists' Society 2011

\section{To the Editor,}

Thoracic paravertebral block (PVB) provides excellent surgical anesthesia as well as postoperative analgesia for thoracic surgeries. ${ }^{1}$ Several ultrasound-guided PVB techniques have been described using either a transverse scan or a parasagittal scan in both the in-plane and the out-of-plane needle insertion approaches. ${ }^{2,3}$ The different approaches rely on the identification of one of two landmarks: 1) the superior costotransverse ligament ${ }^{2}$ (SCTL) or 2) the internal intercostal membrane ${ }^{3}$ (IICM). Learning to scan and identify the SCTL and IICM is possible on volunteers or patients, but, ideally, proficiency of hands-on needle insertion skills is acquired on phantoms prior to patient care given the potential risks of epidural or intrathecal spread, accidental pleural puncture, and pneumothorax associated with thoracic PVB. ${ }^{4}$ It is well known that phantom practice can shorten the learning curve, reduce complications, and result in changes in clinical practice. ${ }^{5}$

F. W. Abdallah, MD · V. W. Chan, MD ( $₫)$

Toronto Western Hospital, University Health Network,

University of Toronto, Toronto, ON, Canada

e-mail: Vincent.Chan@uhn.on.ca

G. Hocking, FANZCA

Sir Charles Gairdner Hospital, School of Medicine and Pharmacology, The University of Western Australia, Perth, WA, Australia
We have designed a pig carcass phantom which can be used to practice ultrasound-guided PVB. A pig weighing $50-60 \mathrm{~kg}$ is purchased from a local abattoir, sacrificed, shaved, decapitated, eviscerated, and washed as per regulations for human consumption. The carcass is then stored in the abattoir fridge at $4^{\circ} \mathrm{C}$ until use (not frozen). The carcass is placed prone for scanning and needle practice. Ultrasound images of the paravertebral space can be obtained using a $12 \mathrm{MHz}$ transducer, and real-time longitudinal parasagittal views show the sonoanatomy of the transverse processes, pleura, and SCTL (Figs. 1, 2). Transverse views show the sonoanatomy of the transverse process, pleura, external intercostal muscle, and internal intercostal membrane. The phantom is useful not only for scanning but also for hands-on practice in needle insertion involving a particularly realistic tactile experience and practice in hand-eye coordination. A successful needle insertion and injection is indicated by downward displacement of the parietal pleura (a hyperechoic line visualized on ultrasound). The addition of methylene blue dye to the injectate permits localization of dye spread along the thoracic paravertebral space after injection, shown by viewing the pleura from the front (Figs. 3, 4). Unintentional pleural puncture is indicated by dye leakage beyond the parietal pleura when inspected from the front. Also, needle track marks are not seen in the flesh after repeated needle insertions.

We recommend the pig carcass phantom for teaching and learning ultrasound-guided thoracic PVB because of the remarkable anatomical similarity of paravertebral space structures in pigs and humans. This is a potentially useful training model to gain competency in scanning and needle insertion for this technique as well as for ultrasound-guided intercostal blocks. 


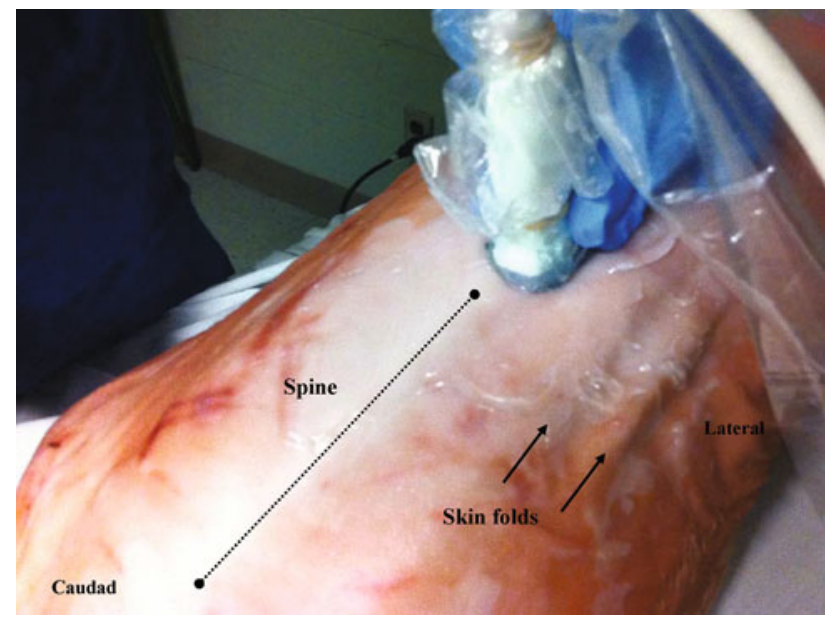

Fig. 1 Parasagittal scan of the porcine paravertebral space

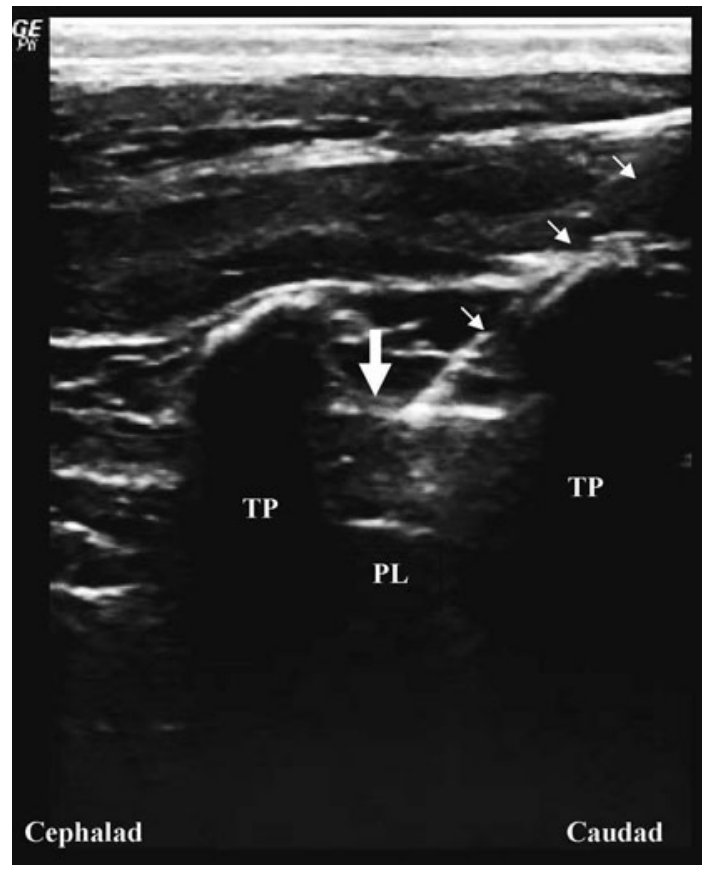

Fig. 2 Real-time needling in the parasagittal approach showing: needle (small arrows), transverse processes (TP), pleura (PL), and superior costotransverse ligament (big arrow)

Acknowledgements We sincerely thank Kris Abbas MD and Cyrus Tse BSc for their fine assistance in preparing and handling the pig specimens for the procedures.

\section{Competing interests None declared.}

Disclosures Dr. Vincent Chan has received equipment support and honoraria from Philips Medical Systems Inc., SonoSite Inc, and GE Medical Inc.

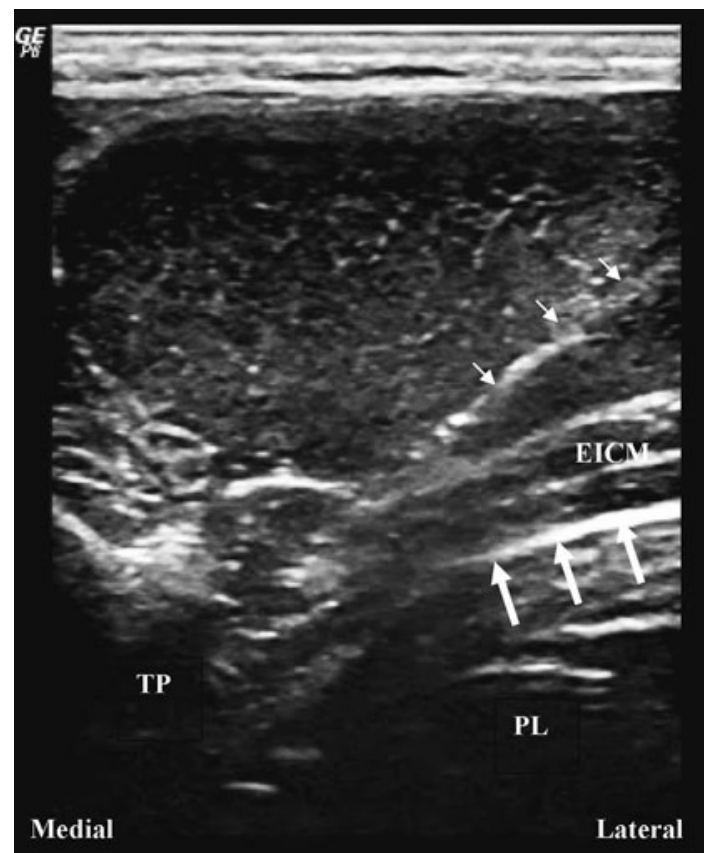

Fig. 3 Real-time needling in the transverse approach showing: needle (small arrows), transverse processes (TP), external intercostal muscle (EICM), pleura (PL), and internal intercostal membrane (large arrows)

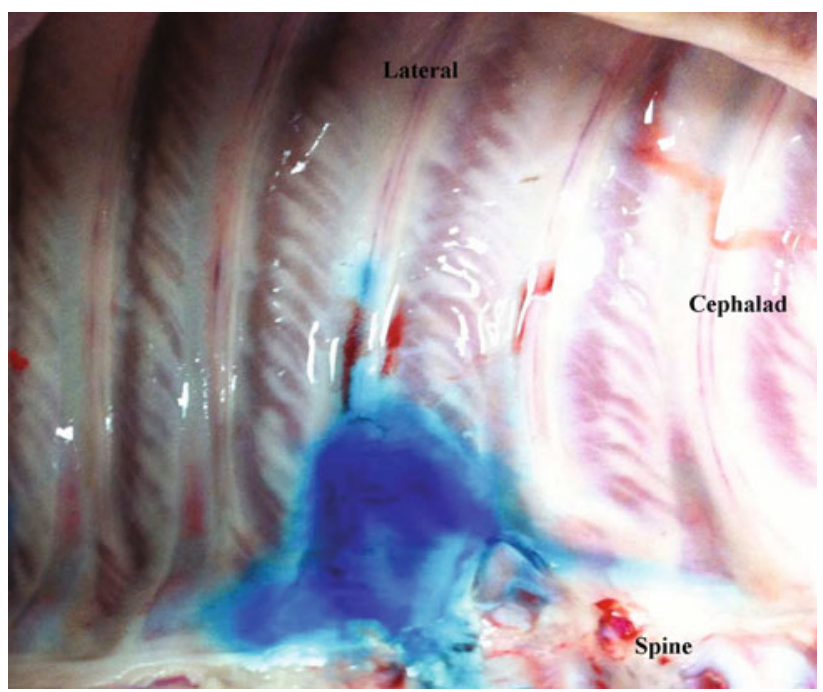

Fig. 4 Successful injection of methylene blue dye showing paravertebral spread in the porcine model

\section{References}

1. Thavaneswaran P, Rudkin GE, Cooter RD, Moyes DG, Perera CL, Maddern GJ. Brief reports: paravertebral block for anesthesia: a systematic review. Anesth Analg 2010; 110: 1740-4.

2. Hara K, Sakura S, Nomura T, Saito Y. Ultrasound guided thoracic paravertebral block in breast surgery. Anaesthesia 2009; 64: 223-5.

3. Shibata $Y$, Nishiwaki $K$. Ultrasound-guided intercostal approach to thoracic paravertebral block. Anesth Analg 2009; 109: 996-7. 
4. Naja Z, Lonnqvist PA. Somatic paravertebral nerve blockade. Incidence of failed block and complications. Anaesthesia 2001; 56: 1184-8.
5. Hocking G, McIntyre $O$. Achieving change in practice by using unembalmed cadavers to teach ultrasound-guided regional anaesthesia. Ultrasound 2011; 19: 31-5. 